\title{
Single-Shot Quantum Nondemolition Detection of Individual Itinerant Microwave Photons
}

\author{
Jean-Claude Besse, 'Simone Gasparinetti, Michele C. Collodo, Theo Walter, Philipp Kurpiers, \\ Marek Pechal, ${ }^{\dagger}$ Christopher Eichler, and Andreas Wallraff \\ Department of Physics, ETH Zurich, CH-8093 Zurich, Switzerland
}

(Received 5 December 2017; revised manuscript received 25 February 2018; published 3 April 2018)

\begin{abstract}
Single-photon detection is an essential component in many experiments in quantum optics, but it remains challenging in the microwave domain. We realize a quantum nondemolition detector for propagating microwave photons and characterize its performance using a single-photon source. To this aim, we implement a cavity-assisted conditional phase gate between the incoming photon and a superconducting artificial atom. By reading out the state of this atom in a single shot, we reach an external (internal) photon-detection fidelity of 50\% (71\%), limited by transmission efficiency between the source and the detector $(75 \%)$ and the coherence properties of the qubit. By characterizing the coherence and average number of photons in the field reflected off the detector, we demonstrate its quantum nondemolition nature. We envisage applications in generating heralded remote entanglement between qubits and for realizing logic gates between propagating microwave photons.
\end{abstract}

DOI: 10.1103/PhysRevX.8.021003

Subject Areas: Quantum Physics, Quantum Information

Single-photon detectors [1] for itinerant fields are a key element in remote entanglement protocols [2], in linear optics quantum computation [3,4], and, in general, in characterizing correlation properties of radiation fields [5]. While such detectors are well established at optical frequencies, their microwave equivalents are still under development, partly because of the much lower photon energy in this frequency band [6]. At microwave frequencies, itinerant fields are typically recorded with linear detection schemes [7], analogous to optical homodyne detection. Such detection can now be realized with high efficiency by employing near-quantum-limited parametric amplifiers [8], and furthermore, it allows for a full tomographic characterization of radiation fields [9]. However, protocols such as entanglement heralding require the intrinsic nonlinearity of a single-photon detector in order to yield high-purity states despite losses between the source and the detector. Such a component has therefore raised interest in the community, leading to a variety of theoretical proposals [10-20], as well as initial experimental demonstrations, in the last decade [21-27].

The first microwave photodetection experiment with superconducting circuits that did not require photons to

\footnotetext{
jbesse@phys.ethz.ch

Present address: Ginzton Laboratory, Stanford University, Stanford, CA 94305, USA.

Published by the American Physical Society under the terms of the Creative Commons Attribution 4.0 International license. Further distribution of this work must maintain attribution to the author(s) and the published article's title, journal citation, and DOI.
}

be stored in high-quality factor cavities [21-23] was based on current biased Josephson junctions [24], but it was destructive and involved a long dead time. Later, systems involving absorption into artificial atoms (and thus destruction) of traveling photons $[25,26]$ were implemented. Very recently, a quantum nondemolition (QND) detection scheme based on a photon-qubit entangling gate, similar in spirit to this work, was implemented using a strong dispersive shift in a 3D cavity [27]. Projective measurements of coherent input states into single-photon Fock states were realized in that work [27].

Here, we demonstrate single-shot QND detection of itinerant single photons in the microwave domain, based on a cavity-assisted controlled phase gate operated between an artificial atom and a propagating photon [28]. We show the unconditional detection of an itinerant wave packet containing a Fock state at the single-photon level.

Our setup consists of a transmon-type superconducting artificial atom coupled to two quarter-wave coplanarwaveguide resonators acting as single-mode cavities [see Fig. 1(a) for a sketch inspired by optical frequency realizations, Fig. 1(b) for a diagram of the circuit realized in the microwave domain, and Appendix A for details]. Both resonators are coupled to a Purcell filter [29], allowing a large bandwidth of readout and detection while protecting the qubit from Purcell decay into the output lines. We tune the first $(|e\rangle)$ to second excited-state $(|f\rangle)$ transition of the transmon, $\omega_{\text {ef }} /(2 \pi)=6135 \mathrm{MHz}$, into resonance with the detector resonator. The transmon anharmonicity is $\alpha /(2 \pi)=-340 \mathrm{MHz}$, such that its ground-state $(|g\rangle)$ to first excited-state transition is at $\omega_{\text {ge }} /(2 \pi)=6475 \mathrm{MHz}$. The transmon is coupled to the detector resonator with an 
(a)

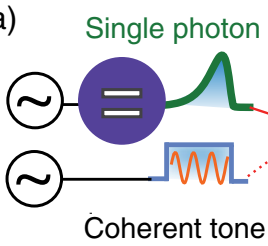

(c)

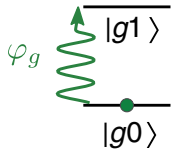

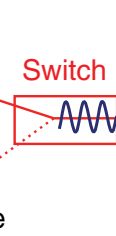

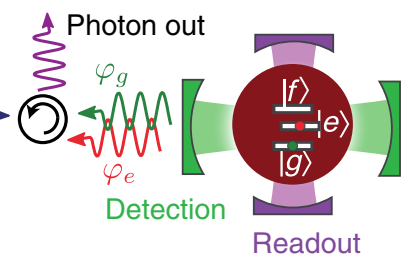

(d)

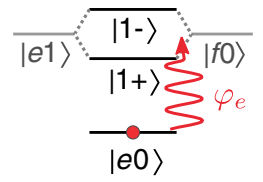

(e) (b)
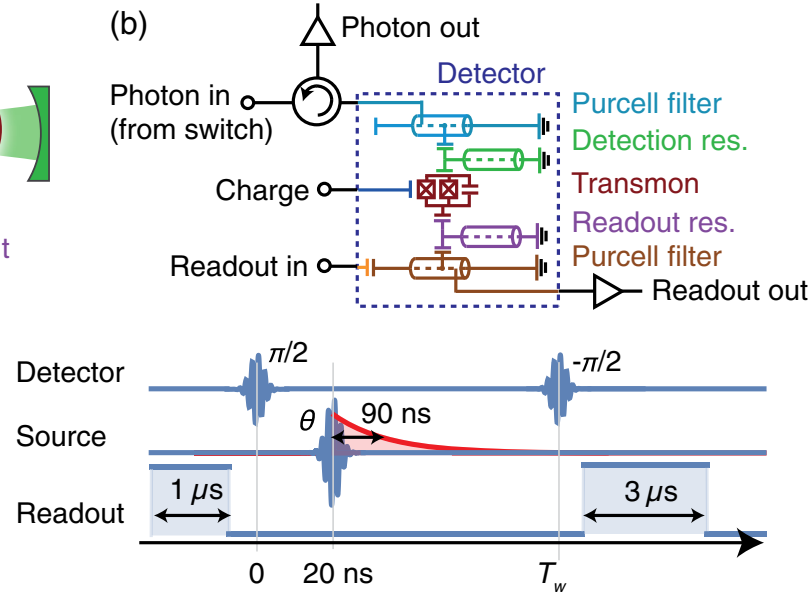

FIG. 1. Principle of quantum nondemolition single-photon detection. (a) Sketch of the setup connecting a source of single photons or a coherent source via a switch and a circulator to the input of the detector. (b) Circuit diagram of the microwave-frequency implementation of the detector chip: A transmon qubit (red) is coupled to the detection resonator (green) and its Purcell filter (light blue), as well as to a readout cavity (purple) and a second Purcell filter (brown). A charge line (dark blue) allows driving of the qubit, and a weakly coupled input port (orange) allows for transmission measurements through the readout resonator. Energy-level diagram of the atom-cavity system when the atom is either in the ground (c) or the excited state (d), contrasting the harmonic ladder of a bare cavity in (c) to the Jaynes-Cummings anharmonic ladder in (d). (e) The pulse scheme consisting of $\pi / 2$ and $-\pi / 2$ pulses applied to the detection qubit, defining the length of the detection time window $T_{w}$, as well as a pulse of Rabi amplitude $\theta$ applied to the source qubit. The temporal mode function of the emitted photon, a decaying exponential with time constant $T_{p}=1 / \Gamma=90 \mathrm{~ns}$, is sketched in red. A pulse applied to the readout resonator is used to measure the state of the detection qubit at the end of the protocol, as well as to preselect the single-shot traces to discard thermal population.

effective linewidth $\kappa /(2 \pi)=19 \mathrm{MHz}$ at rate $g_{0} /(2 \pi)=$ $40 \mathrm{MHz}$. The resulting level diagram is displayed in Figs. 1(c) and 1(d). With the transmon in the ground state $|g\rangle$ [panel (c)], photons impinging on the detector at the resonator frequency acquire a phase $\varphi_{g}=\pi$ as they are reflected. By contrast, with the transmon in $|e\rangle$ [panel (d)], photons of the same frequency are reflected without interacting with the cavity and thus acquire no phase $\left(\varphi_{e}=0\right)$. The readout resonator, at $\omega_{\mathrm{ro}} /(2 \pi)=4800 \mathrm{MHz}$, with effective linewidth $\kappa_{\mathrm{ro}} /(2 \pi)=17 \mathrm{MHz}$ is used to perform high-fidelity, dispersive single-shot readout of the qubit state [30], at a qubit-induced dispersive shift of $\chi_{\mathrm{ro}} /(2 \pi)=17 \mathrm{MHz}$.

We connect the input of our detector to the output of a single-photon source embedded in an on-chip switch [31]. The single-photon source is realized as a transmon strongly coupled to its output port with an emission linewidth of $\Gamma /(2 \pi)=1.77 \mathrm{MHz}$. It emits single photons with a decaying exponential line shape, with time constant $T_{p}=1 / \Gamma=90 \mathrm{~ns}$. The switch is based on a combination of hybrid couplers and tunable resonators. The resonance frequency of the latter is set by static magnetic fields applied to the SQUID loops of the tunable resonators. The switch enables toggling between its two inputs, routing either a coherent tone from a conventional microwave generator or a single-photon wave packet emitted by the source qubit to the input of the detector.

Our protocol for single-photon detection [Fig. 1(e)] begins with a measurement of the transmon state, in order to reject those instances in which the qubit is found to be thermally excited ( $6 \%$ of the total traces were discarded; see Appendix $\mathrm{C}$ for details). We then prepare the transmon in the superposition state $(|g\rangle+|e\rangle) / \sqrt{2}$ using a $\pi / 2$ pulse. This defines the time $t=0$, at which a detection window of length $T_{w}$ begins. Note that $20 \mathrm{~ns}$ later, we emit a photon wave packet in a coherent superposition of vacuum and a single-photon Fock state $|\gamma\rangle=\cos (\theta / 2)|0\rangle+\sin (\theta / 2)|1\rangle$, with a relative weight set by the preparation angle $\theta$ of the Rabi pulse applied to the source qubit. At time $t=T_{w}$, we apply a $-\pi / 2$ pulse to the transmon, effectively completing a Ramsey sequence, and we immediately measure the qubit state.

We first characterize the response of the detector by measuring the phases $\varphi_{e, g}$ [Fig. 2(a)] acquired by a weak coherent tone reflected off the detector input, depending on the state of the transmon [Figs. 1(c) and 1(d)]. We measure the phase difference $\delta \varphi=\varphi_{g}-\varphi_{e}$ by pulsed spectroscopy [Fig. 2(b)] and find $\delta \varphi=\pi$ at the cavity frequency $\omega_{\mathrm{ef}}$, as well as at frequencies $\omega_{\mathrm{ef}} \pm \sqrt{2} g_{0}$ of the qubit-cavity dressed states $|1 \pm\rangle=1 / \sqrt{2}(|e 1\rangle \pm|f 0\rangle)$ [Fig. 1(d)]. In these configurations, a controlled-phase gate is realized between the qubit and a propagating photon. For a definite phase to be acquired by the photon, its spectral bandwidth needs to be smaller than the detector cavity linewidth, $\kappa$. With that condition fulfilled, the gate is independent of the temporal shape of the photon.

The Ramsey sequence displayed in Fig. 1(e) realizes a phase gate on the detection qubit controlled by the presence 


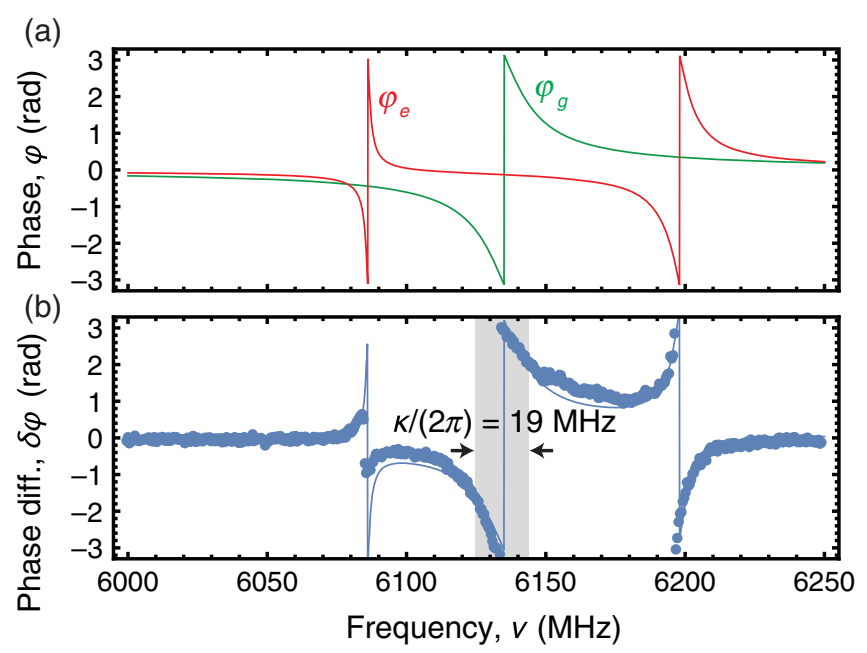

FIG. 2. (a) Expected phase $\varphi_{g}\left(\varphi_{e}\right)$ of a weak coherent signal upon reflection off of the cavity-atom system, when the detection

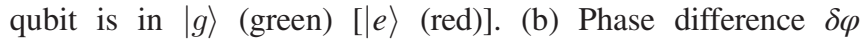
measured in pulsed spectroscopy (blue dots) compared to the calculated value of $\varphi_{g}-\varphi_{e}$ (solid line; see Appendix D). The linewidth $\kappa /(2 \pi)$ of the bare cavity is indicated by the shaded area.

of a photon, implementing the photon-detection process. To characterize the fidelity of the detection process with single photons at the input, we measure the average excitedstate population of the detection qubit as a function of the preparation angle $\theta$ of the photon source [Fig. 3(a), $\left.T_{w}=250 \mathrm{~ns}\right]$. The scaling factor relating the pulse amplitude to the preparation angle for the source is independently calibrated in a Rabi oscillation experiment. The data follow the expected sine-squared dependence, corresponding to the average photon number prepared by the source, with a reduced visibility characterized by the probability $P(e \mid 1)=65.8 \%$ of measuring the detection qubit in the excited state when a photon is emitted and the probability $P(e \mid 0)=5.9 \%$ of measuring the detection qubit in the excited state without emitting a photon. In the context of photon detection, we refer to $P(e \mid 1)$ as the detection efficiency and $P(e \mid 0)$ as the dark count probability. As a performance metric, we define the detection fidelity $F=1-P(g \mid 1)-P(e \mid 0)=P(e \mid 1)-P(e \mid 0)=59.9 \%$ as the difference between detection efficiency and dark count probability. This definition of fidelity is the logical equivalent to the one used to characterize single-shot readout [30].

To gain insight into the sources of errors in the protocol, we extract the detection efficiency and dark count probability vs the duration of detection time window, $T_{w}$ [Fig. 3(b)]. The detection efficiency peaks at an optimal duration of $T_{w} \simeq 300 \mathrm{~ns}$, while the dark count probability monotonically increases with $T_{w}$. A source of errors is the limited coherence $T_{2}^{*}=1.8 \mu$ s of the detection qubit in a Ramsey experiment. A second type of error is due to loss in the components that
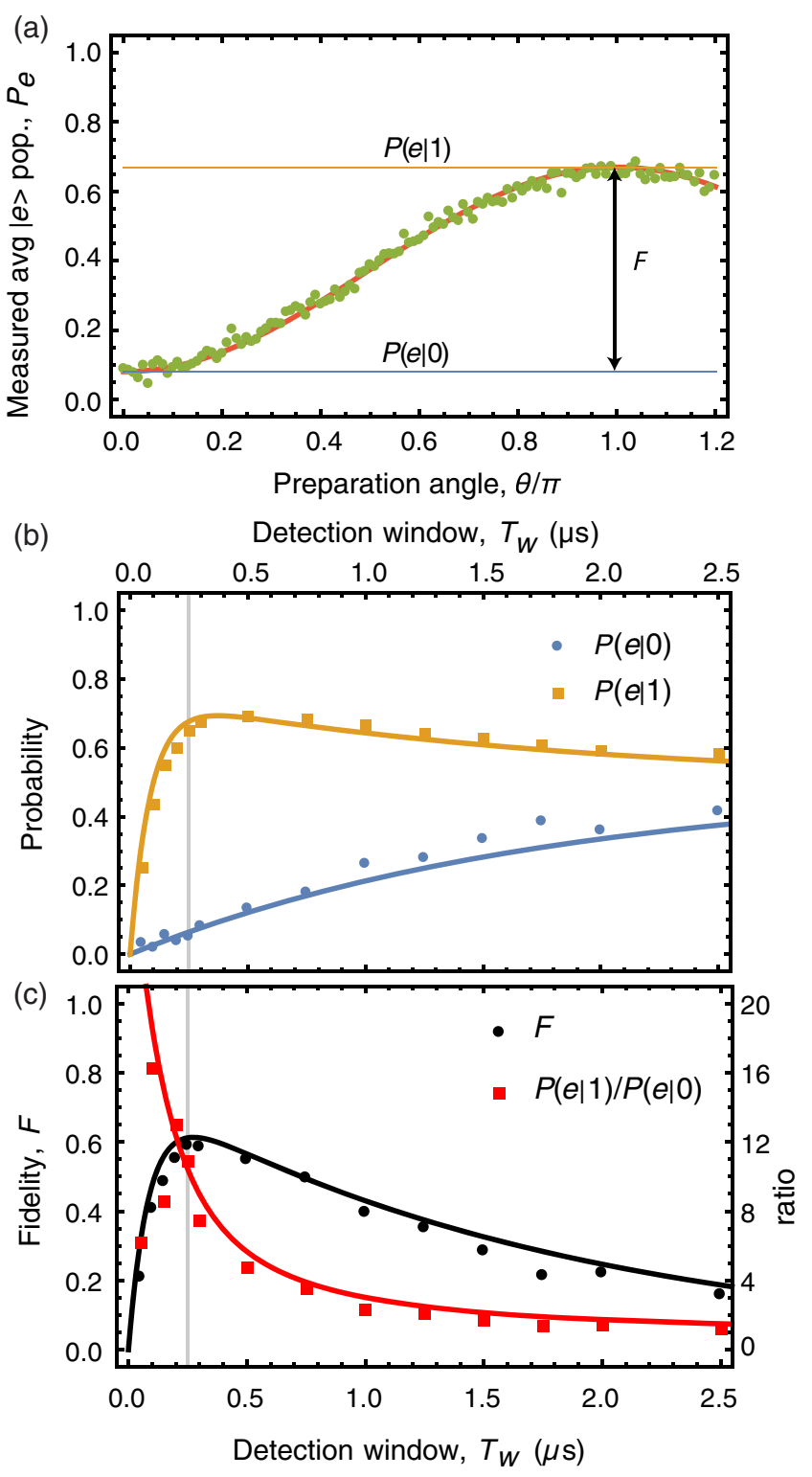

FIG. 3. (a) Measured (averaged readout) excited-state population of the detection qubit (green dots) as a function of the preparation angle $\theta$ of the photon state $|\gamma\rangle$, for detection time window $T_{w}=250 \mathrm{~ns}$. The red line is a fit to the expected dependence, with the detection efficiency $P(e \mid 1)=65.8 \%$ (orange) and the dark count probability $P(e \mid 0)=5.9 \%$ (blue line) as parameters. The fidelity $F=59.9 \%$ is indicated. (b) Dark count probability $P(e \mid 0)$ (blue), photon-detection efficiency $P(e \mid 1)$ (orange), (c) their ratio $P(e \mid 1) / P(e \mid 0)$ (red), and the fidelity $F$ (black) vs length of the detection time window $T_{w}$. Theory lines take into account finite lifetime, losses, and photon line shape (see main text and Appendix D). In the remainder of the paper, the detection time window with $T_{w}=250 \mathrm{~ns}$ is used (vertical gray line).

connect the source to the detector. The measured total loss in the switch, the coaxial cables, and the circulator was found to be approximately 25\% (see Appendix B for calibration measurements using the nonlinear response of the source 
and the detector). From this measurement, we estimate that approximately $75 \%$ of the photons emitted by the source reach the detector, leading to an overall scaling factor independent of $T_{w}$. Finally, for short detection windows, part of the photon envelope is cut off by terminating the protocol with the $-\pi / 2$ pulse prematurely, limiting the detection efficiency. The trends in Fig. 3(b) (solid lines) are quantitatively explained by three sources of errors, which we have identified and characterized independently. For entanglement distribution and other heralded experiments, the ratio $P(e \mid 1) / P(e \mid 0)$ between detection efficiency and dark count probability directly relates to the error rate. We report this ratio, together with the fidelity $F$ vs the length of detection time window $T_{w}$, in Fig. 3(c). While the fidelity peaks at around the same $T_{w}$ as the detection efficiency, the ratio still improves for shorter $T_{w}$ as the dark count probability approaches zero. In our case, this ratio exceeds 10, with significant variations at short $T_{w}$ attributed to fluctuations in the low dark count probability.

The fidelity extracted from Fig. 3(a) refers to an averaged readout. When performing single-shot readout with 100-ns integration time (see Appendix C), we find that the total fidelity of detecting single photons for $T_{w}=250 \mathrm{~ns}$ is $F=50 \%$. This value agrees with the one obtained from the averaged measurements after taking into account the measured $92 \%$ readout fidelity, mainly limited by the transmon decay time $T_{1}=3.0 \mu \mathrm{s}$. The infidelity is due to imperfect detection efficiency, $P(g \mid 1)=37 \%$, and dark count probability, $P(e \mid 0)=13 \%$. After accounting for the calibrated losses before the detector, the internal probability of missing a photon is $P_{\text {in }}(g \mid 1)=16 \%$ (corresponding to a quantum detection efficiency of 0.84 ), so our detector has an internal fidelity of $F_{\text {in }}=1-P_{\text {in }}(g \mid 1)-P(e \mid 0)=71 \%$.

We assess characteristic properties of the detector when detecting fields that contain at most one photon, i.e., in the Hilbert subspace with basis $\{|0\rangle,|1\rangle\}$. This choice is justified noting that we use a source with negligible two-photon emission, as demonstrated by the $g^{(2)}<0.1$ measured in Ref. [31]. As our readout circuit is separated in frequency from the detection, we avoid spurious power input at the detection frequency. We employ a linear amplification chain to measure the average photon number and coherence of the radiation field reflected off of the detector. Those two measurements are sufficient to provide full information about the average density matrix of fields that contain at most one photon [7]. We consider two states of the detector. The "ON" state describes the operation reported so far. In the "OFF" state, we keep the atom in the ground state and detune its frequency to be far off-resonant from the cavity. In both cases, the source emits the same radiation field. We monitor the ensemble-averaged photon number $\left(\left\langle a^{\dagger} a\right\rangle\right.$ moment) and amplitude $(\operatorname{Re}\langle a\rangle$ moment, in the optimized quadrature) by integrating the time traces with a filter matched to the temporal shape of the photon [9]. The results are reported in Fig. 4, together with a
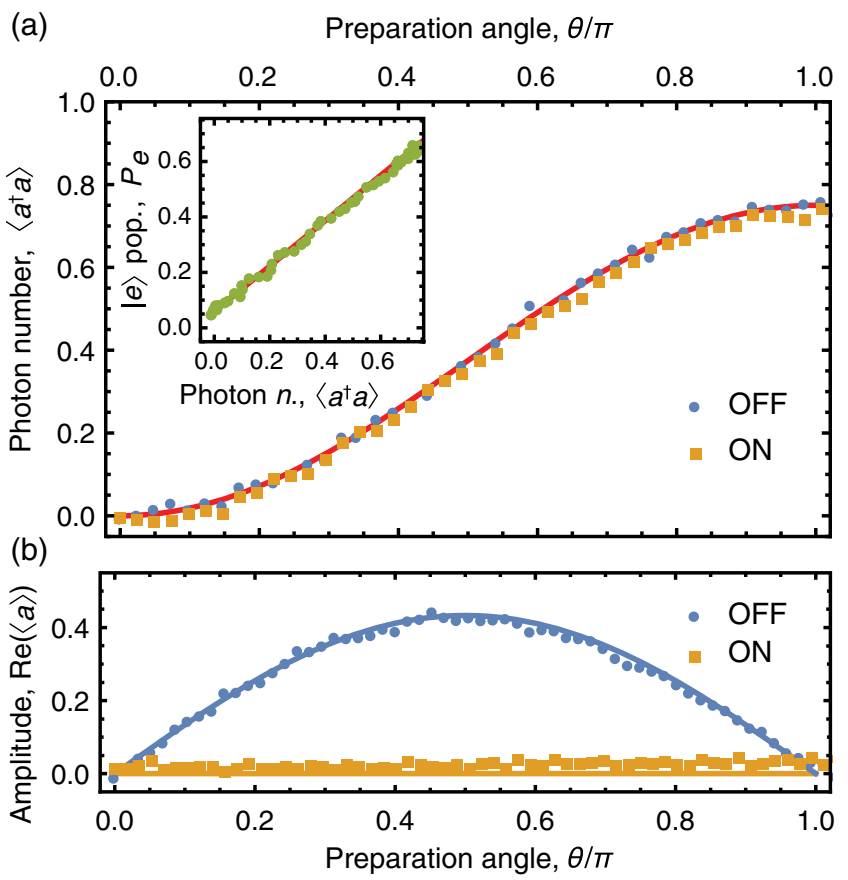

FIG. 4. Nondemolition character of the measurement. (a) Power, reported as photon number $\left\langle a^{\dagger} a\right\rangle$, is conserved, while (b) phase, measured as optimized quadrature $\operatorname{Re}(\langle a\rangle)$, is erased. Solid lines are the expected response of a QND detector. (a, inset) Measurement of the excited-state population $P_{e}$ vs the average outgoing photon number $\left\langle a^{\dagger} a\right\rangle$, with the expected dependence $P_{e}=P(e \mid 0)+F_{\text {in }}\left\langle a^{\dagger} a\right\rangle$.

model. We scale the axes globally by the separately calibrated loss. Up to our measurement accuracy of $2 \%$, we observe no difference in power whether the detection pulse sequence is executed or not [Fig. 4(a)]. Accordingly, we conclude that the detector is performing a nondestructive measurement. At the same time, the phase of the incoming photon state $|\gamma\rangle$ is randomized [Fig. 4(b)], as imposed by quantum mechanics for the conjugate variables $\{n, \phi\}$ in the Hilbert subspace considered. We note that the two states that are left unchanged upon detection are the basis states $|0\rangle(\theta=0)$ and $|1\rangle(\theta=\pi)$. Furthermore, the outcome of the detector measurement is strongly correlated with the outgoing photon power [inset of Fig. 4(a)], such that the probability of our detector clicking is proportional to the single-photon occupation probability. In combination with the nondestructive character of the detection, these observations show that we are performing a QND measurement of the photon number in the single-photon manifold. This is described by the positive-operator valued measure (POVM) with operators $P_{0}=|0\rangle\langle 0|$ for a no-click event and $P_{1}=|1\rangle\langle 1|$ for a click. We measure a small remaining coherence offset in the ON measurement, even without emitting any photons $(\theta=0)$. We ascribe this offset to unintended driving of the $e-f$ transition by the first Ramsey pulse, resulting in the subsequent emission of phase-coherent radiation at the frequency $\omega_{\text {eff }}$. 
In the operation mode presented here (with photons impinging at the bare cavity frequency), we expect that any Fock state with nonvanishing photon number imprints a $\delta \varphi=\pi$ phase shift on the qubit, resulting in a click of the detector. Fock states with $n>0$ are thus expected to be indistinguishable by the detector. On the full Hilbert space of Fock states $|n\rangle, n=0, \ldots, \infty$, the POVM operators corresponding to the no-click and click events are expected to be $P_{0}=|0\rangle\langle 0|$ and $P_{\overline{0}}=\sum_{n=1}^{\infty}|n\rangle\langle n|$. By contrast, detecting photons at one of the dressed-state frequencies in the $n$th manifold and taking advantage of the photonblockade effect generated by the Jaynes-Cummings ladder, we could obtain an operation mode that projectively selects the Fock state with $n$ photons. Here, the POVM operators would be $P_{n}=|n\rangle\langle n|$ and $P_{\bar{n}}=\sum_{m \neq n}|m\rangle\langle m|$. This could be useful in entanglement schemes where a particular Bell state is associated with a definite photon number $n$. The experimental study of the detector response to multiphoton wave packets will be pursued in future work.

We note that, in principle, the protocol can be run continuously with a dead time on the order of the single-shot readout time of $100 \mathrm{~ns}$ by using the readout result as the initial state for the next iteration. One does not need to perform active feedback nor discard the results of an initially excited detection qubit, but instead, one could simply invert the association between the measured qubit state and the presence of a photon.

A clicking detector for itinerant photons in the microwave regime, independent of their temporal shape and with internal fidelity limited by qubit coherence, adds to the circuit QED toolbox for characterizing propagating quantum radiation fields. We have demonstrated single-photon detection with radiation fields at the quantum level, composed of a superposition of vacuum and an $n=1$ photon Fock state. Our device does not internally lose photons upon detection and is built with separate detection and readout lines, which provides easy access to the reflected radiation field. This allows one to take advantage of the nondemolition nature of the detector and use the device as a mediator of photon-photon interactions for allphotonic quantum computation [32-36]. Other applications include heralded entanglement [26] with a high rate, without the need to shape the photons or to perform Bell state analysis [13].

\section{ACKNOWLEDGMENTS}

The authors thank Alexandre Blais, Christian Kraglund Andersen, and Paul Magnard for useful discussions. This work was supported by the European Research Council (ERC) through the Superconducting Quantum Networks (SuperQuNet) project, by the National Center of Competence in Research Quantum Science and Technology (NCCR QSIT) Contract No. 51NF40160591, a research instrument of the Swiss National Science Foundation (SNSF), and by ETH Zurich.

\section{APPENDIX A: SAMPLE FABRICATION AND CABLING}

The sample, shown in Fig. 5, is fabricated on a 4.3-mm $\times$ 7-mm sapphire chip cut along its $c$ plane. All elements except for the qubit are patterned in a 150-nm-thick sputtered niobium film using photolithography and reactive ion etching. The transmon islands and Josephson junctions are fabricated in a separate step using electron-beam lithography and shadow evaporation of aluminum in an electron-beam evaporator. Both the photon-detection device and the single-photon source embedded in an onchip switch [31] are mounted at the base temperature stage $(20 \mathrm{mK})$ of a dilution refrigerator, as shown in the wiring diagram in Fig. 6.

\section{APPENDIX B: CALIBRATION OF PHOTON LOSS}

We distinguish internal detector inefficiencies from photon loss by measuring the attenuation constant between the single-photon source and the detector. This measurement relies on operating both devices as calibrated power sources and comparing the relative power levels detected at room temperature.

First, we employ the photon-blockade effect of the photon source qubit to realize a calibrated power source. We continuously drive the qubit at its transition frequency $\omega_{\mathrm{ge}}$ and measure the power spectral density of the inelastically scattered radiation emitted into the output port (Fig. 7). In the limit of large drive rate $\Omega>\Gamma$, the measured spectrum features characteristic satellite peaks at detunings $\delta \approx \pm \Omega$ relative to the drive frequency [37]. This nonlinear property of the spectrum allows us to calibrate the emitted power $P_{s}=n_{q} \Gamma \hbar \omega_{g e}$ from a global fit of the Mollow triplets. Here, $\Gamma /(2 \pi)=1.77 \mathrm{MHz}$ is the source-qubit

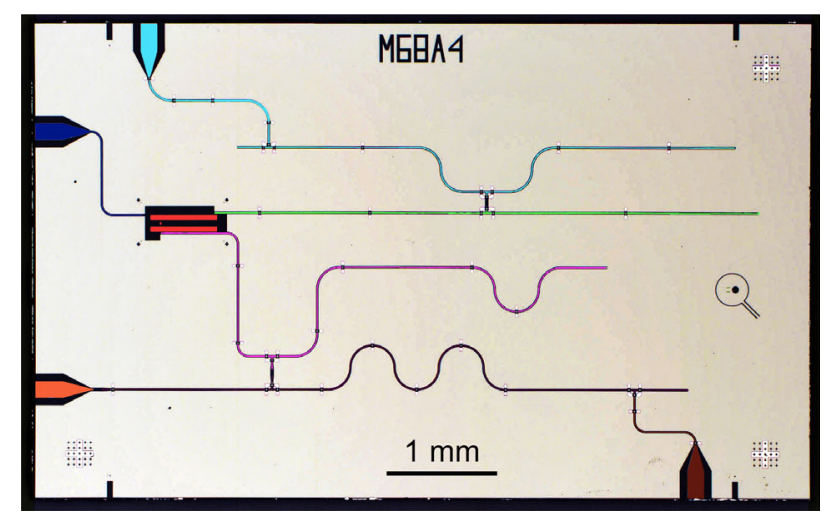

FIG. 5. False color micrograph of the detector sample. A transmon qubit (red) is coupled to the detection cavity (green) and its Purcell filter (light blue), as well as to a readout cavity (purple) and a corresponding Purcell filter (brown). A charge line (dark blue) allows for driving the qubit, and a weakly coupled input port (orange) allows for transmission measurements through the readout resonator. Sapphire is shown in dark gray, and niobium in light gray. 


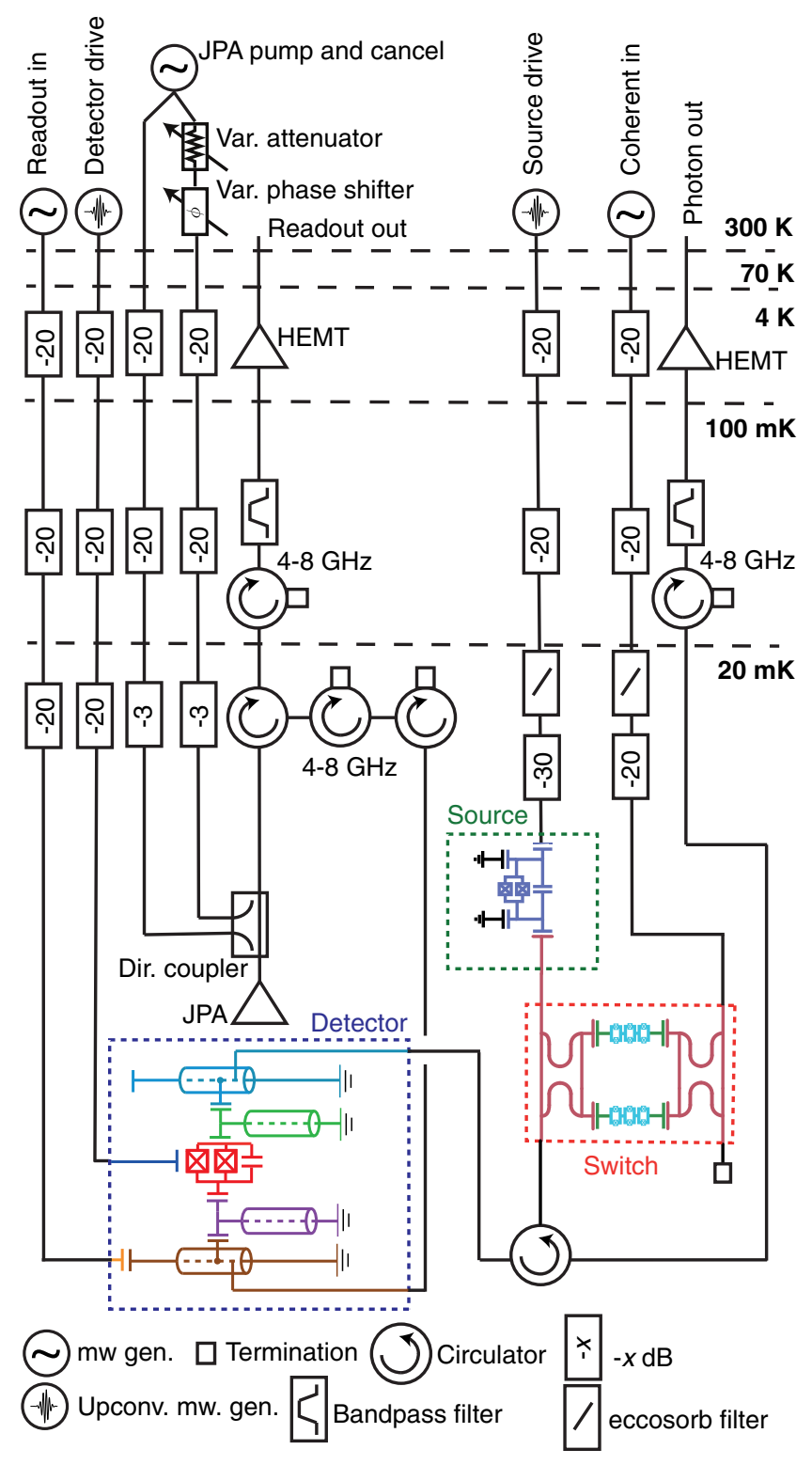

FIG. 6. Scheme of the experimental setup. All microwave lines are inner and outer dc blocked at the room-temperature flange of the cryogenic system. Source and switch are physically on the same sample. The dc cabling for applying external magnetic fields with two coils on the switch sample holder and one coil at the detector is not shown.

linewidth, and $n_{q}$ is the steady-state average excited-state population of the qubit. We note that in the limit of large drive rate $\Omega \gg \Gamma$, the qubit is driven into a mixed state with $n_{q} \approx 1 / 2$. Based on this fit, we obtain the ratio $G_{s}$ of the power detected at room temperature and the power $P_{s}$ emitted from the source. Here, $G_{s}=(1-L) G_{d}$ is composed of the photon loss $L$ from the source to the detector and the effective gain $G_{d}$ from the output of the photon detector to the room-temperature electronics.

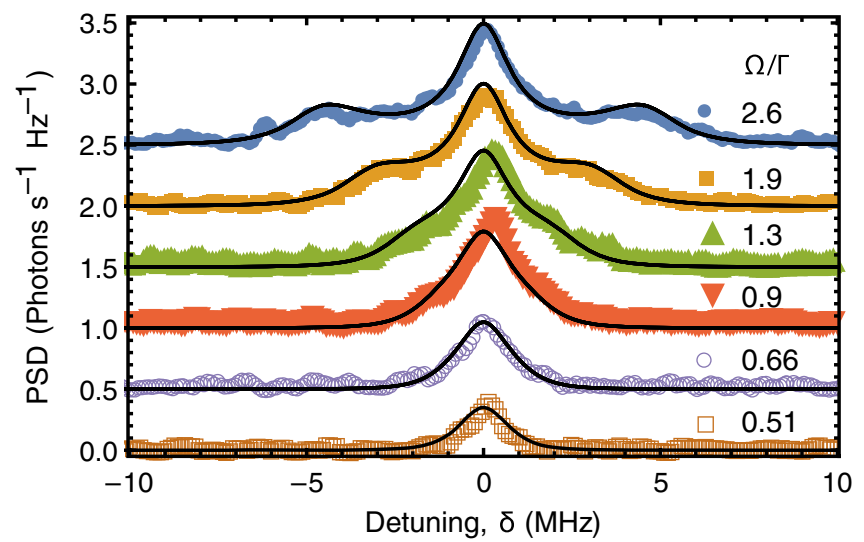

FIG. 7. Measured power spectral density (PSD) of the inelastic scattering of a coherent tone resonant with the source qubit (symbols) for various drive rates $\Omega$ in units of the linewidth $\Gamma$. Solid lines are fits to the data. Individual data sets are offset by 0.5 photons $\mathrm{s}^{-1} \mathrm{~Hz}^{-1}$ for clarity.

To calibrate the gain $G_{d}$, we operate the photon detector as a calibrated power source. For this purpose, we tune the detection qubit to its sweet spot, detuned from the detector resonator by $\Delta /(2 \pi)=\left(\omega_{\text {cav }}-\omega_{\text {ge }}\right) /(2 \pi)=-676 \mathrm{MHz}$, and populate the detector resonator using a coherent tone applied through the second port of the switch. We measure the power-dependent qubit frequency $\omega_{q}$, which decreases linearly with applied power $P_{\text {in }}$ (Fig. 8). The frequency change is due to the ac Stark shift $\Delta_{q}=\omega_{q}-$ $\omega_{q}^{0}=2 \chi n_{p}$ [38]. Independently, we infer the dispersive shift $\chi /(2 \pi)=\alpha g^{2} /(\Delta(\Delta-\alpha)) /(2 \pi)=-2.4 \mathrm{MHz}$ from spectroscopic measurements of the qubit anharmonicity $\alpha /(2 \pi)=-340 \mathrm{MHz}$, and the resonant qubit-cavity coupling $g_{0} /(2 \pi)=40 \mathrm{MHz}$. This yields a calibration for the number of photons $n_{p}$ in the detector resonator. Knowing the effective linewidth $\kappa$ of the cavity, we extract the expected photon power $P_{d}=\kappa n_{p} \hbar \omega_{\text {cav }}$ at the output of the detector. A comparison with the power measured at room temperature yields the effective gain $G_{d}$ of the amplification chain. The loss between the source qubit and the detector resonator is thus estimated as $L_{s-d}=1-$ $G_{s} / G_{d}=0.25$.

We attribute this loss to the following main contributions. First, the circulator placed between the two chips has an insertion loss specified by the manufacturer to be $8 \%$. Second, the finite directivity of the single-pole, doublethrow switch [31] contributes to effective losses by routing about $5 \%$ of the power to its second output, terminated by a $50 \Omega$ load at base temperature. Third, the SMP connectors and female-female bullets used to couple the radiation from a printed circuit board (PCB) to a microwave cable also contribute to a manufacturer-specified insertion loss of $5 \%$. Finally, the attenuation in $50 \mathrm{~cm}$ of $\mathrm{CC} 85 \mathrm{Cu}$ cables connecting the two samples via the circulator amounts to approximately $2 \%$ loss [39]. The total identified sources of 


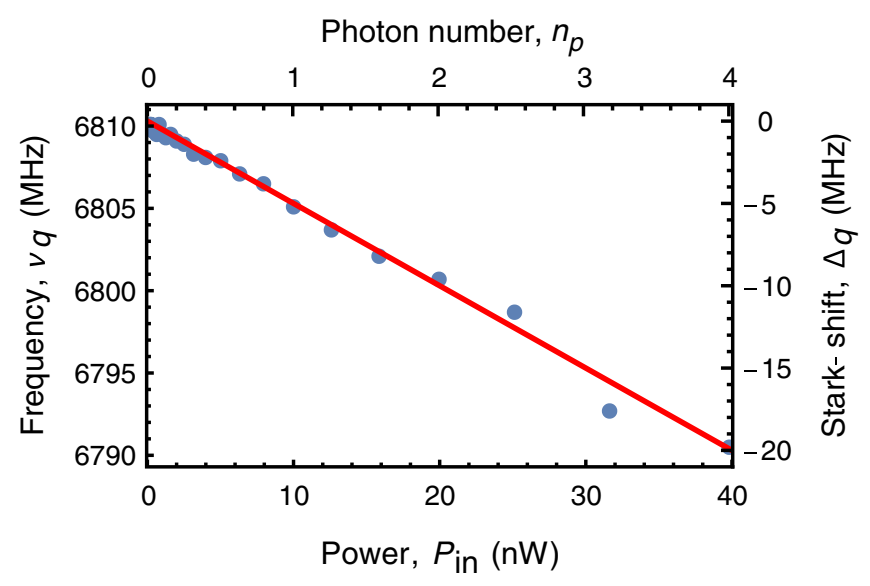

FIG. 8. Stark-shifted frequency $\nu_{q}$ of the detection qubit (blue dots) as a function of the input power $P_{\text {in }}$ at the generator (bottom axis) and the inferred photon number $n_{p}$ in the cavity (top axis). The red curve is a linear fit to the data.

loss add up to approximately $20 \%$. The difference relative to the loss of $25 \%$, extracted from the power detection measurements, could be due to factors not accounted for, such as impedance mismatches or internal losses of components along the detection path, in particular, at wire bonds between samples and PCBs.

\section{APPENDIX C: SINGLE-SHOT READOUT AND DETECTION}

Each experimental sequence starts with a measurement pulse, used to reject approximately $6 \%$ of all measured traces (Fig. 9), in which the qubit was initially found in the excited state. Such instances are due to residual excitations from the previous run and thermal excitations. To realize this preselection measurement, we perform single-shot readout with the methods described in Ref. [30]. We obtain an integrated quadrature amplitude $q$ for each realization, which we compare to a conservatively chosen threshold value to herald the qubit ground state.

To characterize the qubit readout fidelity, we prepare 12500 times the detection-qubit $|g\rangle$ and $|e\rangle$ states. We perform readout in $100 \mathrm{~ns}$ with a gated measurement pulse, obtaining the histograms shown in Fig. 10(a). We extract a readout fidelity of $(91.5 \pm 0.3) \%$. The errors are composed of $P(g \mid e)=(6.3 \pm 0.2) \%$ and $P(e \mid g)=(2.2 \pm 0.1) \%$. The overlap error is below $0.2 \%$.

To obtain the single-shot photon-detection fidelity, we carry out the same readout procedure for 12500 realizations of a single-photon emission in state $|\gamma\rangle=|1\rangle$ or $|\gamma\rangle=|0\rangle$, in both cases performing the detection protocol. The histograms are reported in Fig. 10(b). This corresponds to the singleshot, single-photon-detection fidelity of $F=(49.6 \pm 0.5) \%$ reported in the main text. The infidelity is due to the finite detection efficiency, $P(g \mid 1)=(37.0 \pm 0.4) \%$, dominated

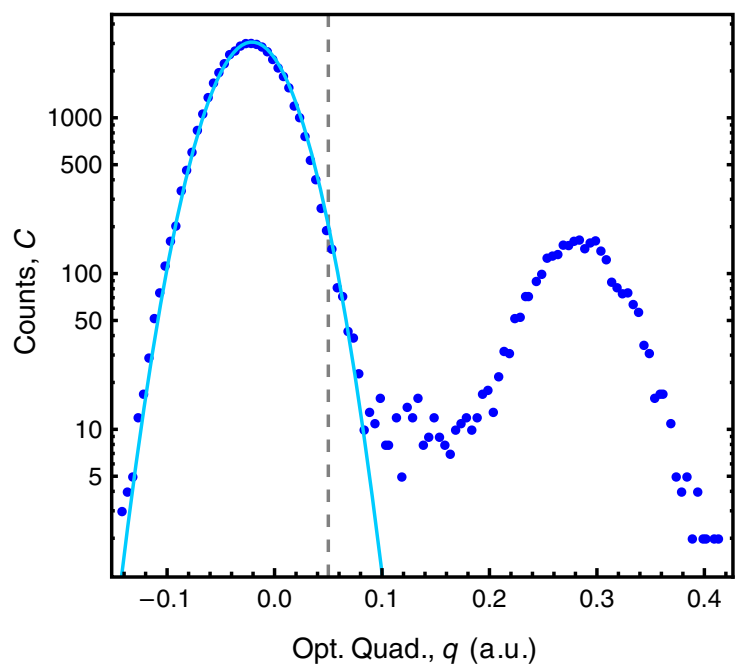

FIG. 9. Histograms of the integrated quadrature amplitude $q$ of the preselection measurement performed to reject initially excited qubit states. The solid line is a fit to a Gaussian. The dashed gray line indicates the selected threshold. Note that $6 \%$ of measurement traces above the threshold are discarded.

by losses between the source and the detector, and the dark count probability, $P(e \mid 0)=(13.4 \pm 0.2) \%$, dominated by the detection-qubit decoherence for the duration of detection window $T_{w}$.

\section{APPENDIX D: MODELING OF PHASE AND FIDELITY}

The phase acquired by a photonic field at frequency $\omega_{p}$ reflected off of a linear cavity with linewidth $\kappa$ is

$$
\varphi_{g}(\delta)=\arg \left(1-\frac{\kappa}{\kappa / 2-i \delta}\right)
$$

where $\delta=\omega_{c}-\omega_{p}$ is the detuning between the cavity frequency $\omega_{c}$ and the photon frequency $\omega_{p}$. In our experimental scheme, this phase shift is induced by the detector when the transmon is in its ground state $|g\rangle$.

In the strong coupling regime where $g_{0} \gg \kappa$, the two states dressed by the Jaynes-Cummings coupling effectively act as two independent modes for a narrowbandwidth photonic field. As a result, the latter acquires the phase

$\varphi_{e}(\delta)=\arg \left(1-\frac{\kappa_{+}}{\kappa_{+} / 2-i\left(\delta+g_{0}\right)}-\frac{\kappa_{-}}{\kappa_{-} / 2-i\left(\delta-g_{0}\right)}\right)$.

One would expect $\kappa_{+}=\kappa_{-}=\kappa / 2$. A frequency-dependent density of states (e.g., due to the Purcell filter) can, however, result in slightly different linewidths for both modes. This scenario happens in our experiment when the transmon is in its excited state $|e\rangle$. Equations (D1) and (D2) 


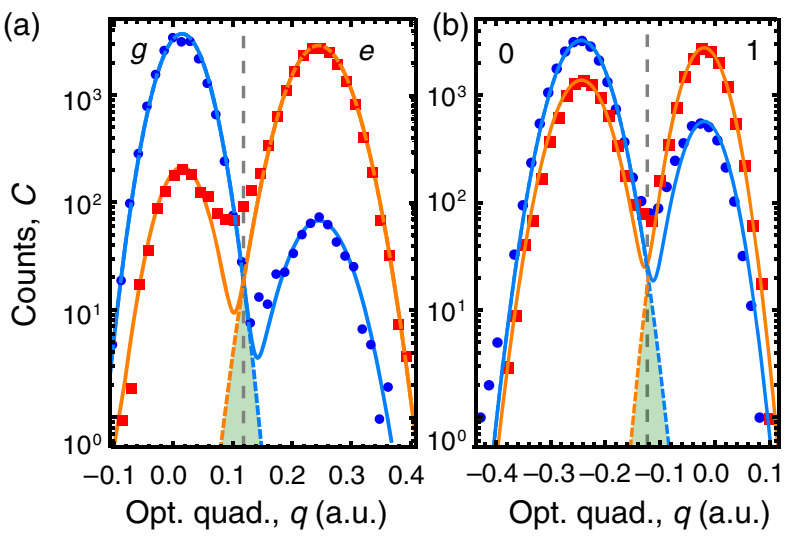

FIG. 10. Single-shot measurements. (a) Histogram of integrated quadrature amplitude $q$ for preparing the state $|g\rangle$ (blue) or $|e\rangle$ (red), as well as (b) for preparing the photon state $|0\rangle$ (blue) or $|1\rangle$ (red). In each panel, the solid lines are fits to a bimodal Gaussian model whose individual components are indicated by dashed lines. The green areas depict the overlap error. The dashed gray line indicates the selected qubit-state threshold.

are plotted in Fig. 2(a), and their difference is plotted in Fig. 2(b).

For the modeling of the dark count probability, we note that our detection scheme performs a Ramsey sequence, such that in the absence of an input photon, one expects to find the excited state with probability

$$
P(e \mid 0)=0.5\left(1-e^{-T_{W} / T_{2}^{*}}\right) .
$$

The detection efficiency is constrained by three effects, as explained in the main text. The first is the success probability $P_{\text {Ramsey }}=0.5\left(1+e^{-T_{W} / T_{2}^{*}}\right)$ of the Ramsey sequence. The second is the probability $1-P_{\text {loss }}=1-L$ that a photon emitted by the source reaches the detector. The third is the fraction $P_{\text {shape }}=\int_{0}^{T_{W}} 1 / T_{p} e^{-t / T_{p}} \mathrm{~d} t=$ $1-e^{-T_{W} / T_{p}}$ of the photon power that is contained within the detection time window of length $T_{W}$.

Two possible configurations lead to the detector clicking when a photon is prepared: (i) Either the Ramsey sequence succeeds, and the emitted photon is reflected from the detector within the detection time window, or (ii) the Ramsey sequence fails, and the emitted photon is either absorbed before the detector or arrives outside of the detection time window. The sum leads to

$$
\begin{aligned}
P(e \mid 1)= & P_{\text {Ramsey }}\left(1-P_{\text {loss }}\right) P_{\text {shape }} \\
& +\left(1-P_{\text {Ramsey }}\right)\left[1-\left(1-P_{\text {loss }}\right) P_{\text {shape }}\right] \\
= & 0.5\left(1+e^{-T_{W} / T_{2}^{*}}\right)(1-L)\left(1-e^{-T_{W}} / T_{p}\right) \\
& +0.5\left(1-e^{-T_{W} / T_{2}^{*}}\right)\left[1-(1-L)\left(1-e^{-T_{W}} / T_{p}\right)\right] .
\end{aligned}
$$

Equations (D4) and (D3) are plotted in Fig. 3(b). Their difference, defining the fidelity $F=P(e \mid 1)-P(e \mid 0)$, and their ratio $P(e \mid 1) / P(e \mid 0)$ are plotted in Fig. 3(c).

[1] R. H. Hadfield, Single-Photon Detectors for Optical Quantum Information Applications, Nat. Photonics 3, 696 (2009).

[2] C. W. Chou, H. de Riedmatten, D. Felinto, S. V. Polyakov, S. J. van Enk, and H. J. Kimble, Measurement-Induced Entanglement for Excitation Stored in Remote Atomic Ensembles, Nature (London) 438, 828 (2005).

[3] P. Kok, W. J. Munro, K. Nemoto, T. C. Ralph, J. P. Dowling, and G. J. Milburn, Linear Optical Quantum Computing with Photonic Qubits, Rev. Mod. Phys. 79, 135 (2007).

[4] E. Knill, R. Laflamme, and G. J. Milburn, A Scheme for Efficient Quantum Computation with Linear Optics, Nature (London) 409, 46 (2001).

[5] R. J. Glauber, Photon Correlations, Phys. Rev. Lett. 10, 84 (1963).

[6] X. Gu, A. F. Kockum, A. Miranowicz, Y.-X. Liu, and F. Nori, Microwave Photonics with Superconducting Quantum Circuits, Phys. Rep. 718, 1 (2017).

[7] C. Eichler, D. Bozyigit, and A. Wallraff, Characterizing Quantum Microwave Radiation and Its Entanglement with Superconducting Qubits Using Linear Detectors, Phys. Rev. A 86, 032106 (2012).

[8] M. A. Castellanos-Beltran and K. W. Lehnert, Widely Tunable Parametric Amplifier Based on a Superconducting Quantum Interference Device Array Resonator, Appl. Phys. Lett. 91, 083509 (2007).

[9] C. Eichler, D. Bozyigit, C. Lang, L. Steffen, J. Fink, and A. Wallraff, Experimental State Tomography of Itinerant Single Microwave Photons, Phys. Rev. Lett. 106, 220503 (2011).

[10] G. Romero, J. J. García-Ripoll, and E. Solano, Microwave Photon Detector in Circuit QED, Phys. Rev. Lett. 102, 173602 (2009).

[11] F. Helmer, M. Mariantoni, E. Solano, and F. Marquardt, Quantum Nondemolition Photon Detection in Circuit QED and the Quantum Zeno Effect, Phys. Rev. A 79, 052115 (2009).

[12] B. Peropadre, G. Romero, G. Johansson, C. M. Wilson, E. Solano, and J. J. García-Ripoll, Approaching Perfect Microwave Photodetection in Circuit QED, Phys. Rev. A 84, 063834 (2011).

[13] D. Witthaut, M. D. Lukin, and A. S. Sørensen, Photon Sorters and QND Detectors Using Single Photon Emitters, Europhys. Lett. 97, 50007 (2012).

[14] M. T. Manzoni, F. Reiter, J. M. Taylor, and A. S. Sørensen, Single-Photon Transistor Based on Superconducting Systems, Phys. Rev. B 89, 180502 (2014).

[15] B. Fan, G. Johansson, J. Combes, G. J. Milburn, and T. M. Stace, Non-absorbing High-Efficiency Counter for Itinerant Microwave Photons, Phys. Rev. B 90, 035132 (2014).

[16] S. R. Sathyamoorthy, L. Tornberg, A. F. Kockum, B. Q. Baragiola, J. Combes, C. M. Wilson, T. M. Stace, and G. Johansson, Quantum Nondemolition Detection of a Propagating Microwave Photon, Phys. Rev. Lett. 112, 093601 (2014). 
[17] K. Koshino, K. Inomata, Z. Lin, Y. Nakamura, and T. Yamamoto, Theory of Microwave Single-Photon Detection Using an Impedance-Matched $\lambda$ System, Phys. Rev. A 91, 043805 (2015).

[18] S. R. Sathyamoorthy, T. Stace, and G. Johansson, Detecting Itinerant Single Microwave Photons, C.R. Phys. 17, 756 (2016).

[19] O. Kyriienko and A. S. Sorensen, Continuous-Wave SinglePhoton Transistor Based on a Superconducting Circuit, Phys. Rev. Lett. 117, 140503 (2016).

[20] K. Koshino, Z. Lin, K. Inomata, T. Yamamoto, and Y. Nakamura, Dressed-State Engineering for Continuous Detection of Itinerant Microwave Photons, Phys. Rev. A 93, 023824 (2016).

[21] S. Gleyzes, S. Kuhr, C. Guerlin, J. Bernu, S. Deleglise, U. B. Hoff, M. Brune, J.-M. Raimond, and S. Haroche, Quantum Jumps of Light Recording the Birth and Death of a Photon in a Cavity, Nature (London) 446, 297 (2007).

[22] B. R. Johnson, M. D. Reed, A. A. Houck, D. I. Schuster, L. S. Bishop, E. Ginossar, J. M. Gambetta, L. DiCarlo, L. Frunzio, S. M. Girvin, and R. J. Schoelkopf, Quantum Non-demolition Detection of Single Microwave Photons in a Circuit, Nat. Phys. 6, 663 (2010).

[23] P. J. Leek, M. Baur, J. M. Fink, R. Bianchetti, L. Steffen, S. Filipp, and A. Wallraff, Cavity Quantum Electrodynamics with Separate Photon Storage and Qubit Readout Modes, Phys. Rev. Lett. 104, 100504 (2010).

[24] Y.-F. Chen, D. Hover, S. Sendelbach, L. Maurer, S. T. Merkel, E. J. Pritchett, F. K. Wilhelm, and R. McDermott, Microwave Photon Counter Based on Josephson Junctions, Phys. Rev. Lett. 107, 217401 (2011).

[25] K. Inomata, Z. Lin, K. Koshino, W. D. Oliver, J. Tsai, T. Yamamoto, and Y. Nakamura, Single Microwave-Photon Detector Using an Artificial ג-Type Three-Level System, Nat. Commun. 7, 12303 (2016).

[26] A. Narla, S. Shankar, M. Hatridge, Z. Leghtas, K. M. Sliwa, E. Zalys-Geller, S. O. Mundhada, W. Pfaff, L. Frunzio, R. J. Schoelkopf, and M. H. Devoret, Robust Concurrent Remote Entanglement Between Two Superconducting Qubits, Phys. Rev. X 6, 031036 (2016).

[27] S. Kono, K. Koshino, Y. Tabuchi, A. Noguchi, and Y. Nakamura, Quantum Non-demolition Detection of an Itinerant Microwave Photon, arXiv:1711.05479.

[28] L.-M. Duan and H. J. Kimble, Scalable Photonic Quantum Computation through Cavity-Assisted Interactions, Phys. Rev. Lett. 92, 127902 (2004).
[29] M. D. Reed, B. R. Johnson, A. A. Houck, L. DiCarlo, J. M. Chow, D. I. Schuster, L. Frunzio, and R. J. Schoelkopf, Fast Reset and Suppressing Spontaneous Emission of a Superconducting Qubit, Appl. Phys. Lett. 96, 203110 (2010).

[30] T. Walter, P. Kurpiers, S. Gasparinetti, P. Magnard, A. Potocnik, Y. Salathé, M. Pechal, M. Mondal, M. Oppliger, C. Eichler, and A. Wallraff, Rapid, High-Fidelity, SingleShot Dispersive Readout of Superconducting Qubits, Phys. Rev. Applied 7, 054020 (2017).

[31] M. Pechal, J.-C. Besse, M. Mondal, M. Oppliger, S. Gasparinetti, and A. Wallraff, Superconducting Switch for Fast On-Chip Routing of Quantum Microwave Fields, Phys. Rev. Applied 6, 024009 (2016).

[32] B. Hacker, S. Welte, G. Rempe, and S. Ritter, A PhotonPhoton Quantum Gate Based on a Single Atom in an Optical Resonator, Nature (London) 536, 193 (2016).

[33] R. Kokkoniemi, T. Ollikainen, R. E. Lake, S. Saarenpaa, K. Y. Tan, J. I. Kokkala, C. B. Dag, J. Govenius, and M. Mottonen, Quantum Gates for Propagating Microwave Photons, arXiv:1703.02241.

[34] K. Koshino, K. Inomata, Z. R. Lin, Y. Tokunaga, T. Yamamoto, and Y. Nakamura, Theory of Deterministic Entanglement Generation between Remote Superconducting Atoms, Phys. Rev. Applied 7, 064006 (2017).

[35] G.-Y. Wang, Q. Liu, H.-R. Wei, T. Li, Q. Ai, and F.-G. Deng, Universal Quantum Gates for Photon-Atom Hybrid Systems Assisted by Bad Cavities, Sci. Rep. 6, 24183 (2016).

[36] M. Hua, M.-J. Tao, and F.-G. Deng, Fast Universal Quantum Gates on Microwave Photons with All-Resonance Operations in Circuit QED, Sci. Rep. 5, 9274 (2015).

[37] C. Lang, D. Bozyigit, C. Eichler, L. Steffen, J. M. Fink, A. A. Abdumalikov Jr., M. Baur, S. Filipp, M. P. da Silva, A. Blais, and A. Wallraff, Observation of Resonant Photon Blockade at Microwave Frequencies Using Correlation Function Measurements, Phys. Rev. Lett. 106, 243601 (2011).

[38] D. I. Schuster, A. Wallraff, A. Blais, L. Frunzio, R.-S. Huang, J. Majer, S. M. Girvin, and R. J. Schoelkopf, AC-Stark Shift and Dephasing of a Superconducting Qubit Strongly Coupled to a Cavity Field, Phys. Rev. Lett. 94, 123602 (2005).

[39] P. Kurpiers, T. Walter, P. Magnard, Y. Salathe, and A. Wallraff, Characterizing the Attenuation of Coaxial and Rectangular Microwave-Frequency Waveguides at Cryogenic Temperatures, Eur. Phys. J. Quantum Techn. 4, 8 (2017). 